\title{
Chiacchiere in sala d'attesa: una dializzata in Dermatologia
}

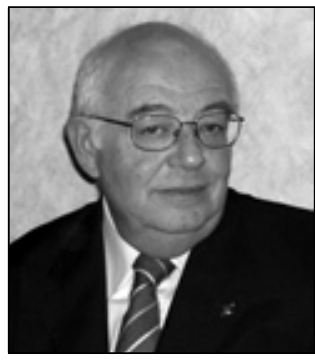

Dr. Bruno Bertone bruno.bertone@virgilio.it

Quando arrivo nell'ambulatorio ospedaliero di Dermatologia c'è una signora in attesa, saluto, mi seggo. Nel mio caso si tratta di una visita di routine, un controllo della psoriasi che da anni ormai mi tiene compagnia. Dopo qualche minuto arriva una seconda signora, saluta e si accomoda. Chiameremo la persona appena arrivata Seconda signora, mentre chiameremo Prima signora quella già presente al mio arrivo.

- Seconda signora si rivolge a Prima signora: «è tanto che aspetta?».

- Prima signora: «oggi sembra che la facciano lunga, c’è dentro una signora con un bambino».

Seconda signora guarda l'orologio tre volte in meno di un minuto, rilevo la cosa. È improbabile che Seconda signora debba rientrare al lavoro, pare aver superato l'età lavorativa già da qualche anno, sembra comunque aver fretta. Alla quarta occhiata all'orologio cedo alla curiosità e chiedo a Seconda signora: «ha fretta signora?». Non è che io abbia un personale interesse ai programmi di Seconda signora, mi interessa solo sapere se la mia lettura del suo guardare l'orologio è corretta.

- Seconda signora: «non è che abbia fretta è che poi devo andare a fare la dialisi e l'appuntamento sarebbe tra mezzora».

A quella risposta mi offro di farla passare prima di me e aggiungo che può telefonare in dialisi per avvisarli che potrebbe ritardare.

Seconda signora mi ringrazia ma dice che non occorre né che la faccia passare, né che lei telefoni in dialisi per- ché: "tanto quando arrivo mi mettono su". Sono spiazzato dall'espressione che impiega, non capisco se sia gergo del reparto di dialisi o gergo personale di Seconda signora.

- Prima signora si riscuote alla parola dialisi e, rivolta a Seconda signora, chiede: «ma allora lei è sempre qui in ospedale?».

- Seconda signora risponde di essere in ospedale per tre giorni a settimana e sorridendo aggiunge: «oggi però è una giornata speciale perché mi faccio dermatologia e dialisi».

Prima signora chiede, a questo punto, se abbia anche un problema dermatologico e Seconda signora risponde che avendo prurito da qualche giorno si è fatta prescrivere una visita dermatologica dal suo medico di famiglia.

- Prima signora riflette e poi: «certo che la visita dermatologica, poi la dialisi andrà a finire che arriva a casa tardi per la cena?».

- Seconda signora: «per la cena non ho problemi, sono sola apro la scatola del formaggio e mangio pane e toma*, è la mia cena di quando è tardi per cucinare, mi piace il formaggio».

Il discorso si fa interessante e non mi riesce di starne fuori, infatti faccio anch'io una domanda a Seconda signora: «ho sentito che chi fa la dialisi dovrebbe seguire una dieta, è una cosa che le pesa?».

- Seconda signora: «prima di entrare in dialisi mi pesava la dieta, adesso che sono in dialisi posso mangiare praticamente tutto quello che voglio, anche se con il formaggio forse esagero un pochino!».

- Prima signora interviene: «dice che esagera col formaggio perché poi ha difficoltà a digerire?».

- Seconda signora: «no lo digerisco benissimo, è solo perché la dietista mi ha detto di non mangiarne molto».

Intervengo con una seconda domanda: «... e le pesa prendere le molte medicine che mi dicono vengano prescritte ai dializzati?».

- Seconda signora: «non sono molte, sono troppe, ma io

${ }^{*}$ Formaggio fresco ottenuto da latte di capra, vacca o pecora. 
non intendo avvelenarmi, mi faccio uno sconto, non le prendo tutte, tanto quella che conta è la dialisi».

Sto pensando che posso intervistare a ruota libera una dializzata, mi interesserebbe sapere come si raffigura la propria malattia e cosa pensa della terapia sostitutiva. Sarebbe fantastico se dal colloquio emergesse la sua rappresentazione di malattia (1).

Il clima di apertura al dialogo viene interrotto dall'arrivo di una conoscente di Seconda signora, le due donne si salutano calorosamente e subito iniziano a parlare fitto fitto tra di loro.

Ho perso la mia occasione, anche perché in men che non si dica è il mio turno per entrare in sala visita.

\section{Analisi dell'accaduto in sala d'attesa e ipotesi}

Come possiamo interpretare quanto è stato detto? Possiamo farci un'idea di come Seconda signora aderisca alla dieta e alla terapia prescritta? Possiamo ipotizzare una sua rappresentazione di malattia?

Le dichiarazioni di Seconda signora riguardano:

La dieta

- mi pesava prima di entrare in dialisi

- ora (che sono in dialisi) non mi pesa più

- mangio praticamente quello che voglio
- il formaggio è la mia cena quando rientro tardi

- forse esagero un pochino col formaggio.

La terapia

- le medicine sono troppe

- mi faccio uno sconto (quantitativo o qualitativo?)

- non prendo tutte le medicine ma quella che conta è la dialisi

- non intendo avvelenarmi con le medicine.

Avendo elencato le dichiarazioni di Seconda signora non è possibile non fare ipotesi sulla sua presenza dal dermatologo:

Dichiara di mangiare formaggio spesso.

Dice che forse esagera col formaggio.

Che sia il troppo fosforo assunto a causare il prurito? $(2,3)$.

Che il prurito sia conseguente a un problema dermatologico senza alcuna relazione con la sua insufficienza renale?

Se il problema di Seconda signora fosse esclusivamente di tipo dermatologico verrebbe affrontato e possibilmente risolto dallo specialista. Se, invece, il prurito fosse dovuto a un'eccessiva assunzione di fosforo allora sarebbe necessario un cambiamento di abitudini alimentari e accanto a queste una maggior adesione alla terapia, dato

TABELLA I - RAPPRESENTAZIONE DI MALATTIA SECONDO M.A. DIEFENBACH (1)

\begin{tabular}{|l|l|}
\hline \multicolumn{1}{|c|}{ Componenti } & \multicolumn{1}{c|}{ Descrizione } \\
\hline Identità & $\begin{array}{l}\text { Etichettatura della malattia ed idee } \\
\text { personali su segni e sintomi. }\end{array}$ \\
\hline Causa & $\begin{array}{l}\text { Convinzioni personali sulle cause } \\
\text { di malattia (Es.: genetiche, dovute } \\
\text { allo stile di vita, altro) }\end{array}$ \\
\hline Cronologia & $\begin{array}{l}\text { Opinione personale sul tipo di } \\
\text { malattia: acuta o cronica. }\end{array}$ \\
\hline Conseguenze & $\begin{array}{l}\text { Opinioni sull'impatto di malattia in } \\
\text { termini di esperienze personali, } \\
\text { impegno economico, stress emotivo, } \\
\text { ruolo sociale, etc. }\end{array}$ \\
\hline Controllo/Trattamento & $\begin{array}{l}\text { Convinzioni personali sulla } \\
\text { comprensione dello stimolo ad } \\
\text { interventi individuali e degli } \\
\text { operatori sanitari. }\end{array}$ \\
\hline
\end{tabular}


che la paziente stessa dichiara di "farsi uno sconto" sui farmaci.

E sicuramente importante sapere quale sia l'adesione alla terapia e alla dieta delle persone dializzate (4): in un certo numero di casi la terapia di supporto prescritta rimane nelle intenzioni del curante, ma il paziente non la assume del tutto o non come prescritto $(7,8)$.

Se fosse possibile, per il curante, conoscere la rappresentazione di malattia dei pazienti potrebbe fare una valida ipotesi sulla loro adesione alla terapia e sul loro equilibrio emotivo (5).

Già ma cos'è la rappresentazione di malattia e per quale motivo sarebbe opportuno che il personale la conoscesse? $(1,6)$.

Secondo A. Diefenbach la rappresentazione di malattia risulterebbe dalle componenti elencate nella Tabella I.

Da quanto ha detto Seconda signora appare assai difficoltoso anche solo fare ipotesi su tutte le componenti della sua rappresentazione di malattia, si può provare, comunque a ipotizzare alcune delle sue idee sulla malattia e sul trattamento.

Iniziamo a considerare la dieta. Seconda signora afferma che la dieta le pesava prima di entrare in dialisi ma ora non più perché sceglie i cibi più liberamente. Dalla sua affermazione si potrebbe dedurre che le raccomandazioni dietetiche vengano percepite come meno limitanti. In effetti però la dietista le ha detto di non mangiare troppo formaggio: dovremmo sapere troppo rispetto a che quantità (giornaliera/settimanale) consentita! Le persone (e noi tra loro) hanno spesso la tendenza ad esprimere le quantità in modo generico: poco, molto, troppo. Si dovrebbe sempre precisare, espressioni di quantità generiche sono individuali: ciò che è poco per l'uno può essere molto per l'altro. Con ogni probabilità la dietista ha precisato a Seconda signora le quantità di formaggio che le sono consentite, la paziente ne mangia quantità maggiori e minimizza dicendo: “... forse esagero un pochino”. Se si evidenziasse che la dieta di Seconda signora non è in linea con le esigenze si dovrebbe intervenire per correggere l'abitudine alimentare. L'intervento su un paziente che non aderisce a una prescrizione non deve mai essere giudicante(9). Il medico e anche l'operatore dovrebbero intervenire sempre supportando e mai redarguendo o stigmatizzando un comportamento. L'obiettivo deve essere far perseguire al paziente il comportamento corretto non dare un giudizio negativo su di un comportamento non corretto (9).

Passando alla terapia possiamo rilevare come Seconda signora dica che le medicine (da assumere) sono troppe, ma lei si fa uno sconto: quindi esclude uno/più farmaci oppure auto-riduce il numero di somministrazioni (ancor peggio potrebbe fare entrambe le cose in contemporanea). La paziente aggiunge che non intende avvelenarsi con tutte quelle medicine. Questa dichiarazione può far pensare che il suo atteggiamento nei confronti dei farmaci sia di tipo difensivo: "le medicine avvelenano ed io ne assumo una quantità che valuto accettabile". L'atteggiamento difensivo genera un comportamento di non adesione alla terapia. Ad esempio, se uno dei farmaci scartati da Seconda signora fosse un chelante del fosforo potrebbe essere alla base dell'aumento dei livelli di fosforo e quindi del sintomo prurito.

Seconda signora sembra voler giustificare la sua solo parziale adesione alla terapia dicendo che poi, alla fin fine, quella che conta è la dialisi.

Quest'idea, che la paziente parrebbe esprimere, indicherebbe anche una graduatoria di valori: alta quella assegnata alla procedura dialitica, più bassa quella assegnata alla terapia farmacologica e alla dieta che, per tale motivo, possono essere adattate/ridotte, in base a presunte esigenze personali.

Un intervento educativo a favore della paziente dovrebbe avere quale obiettivo il ripristino del valore da assegnare alla dialisi, alla terapia farmacologica e alla dieta. Dopo un intervento educativo efficace Seconda signora dovrebbe esprimere così la sua nuova idea: "La terapia sostitutiva è un insieme di dialisi, farmaci e dieta che nel loro insieme consentono il controllo della malattia renale".

Non è stato possibile neppure intravedere il quadro di rappresentazione di malattia di Seconda signora, abbiamo comunque ipotizzato alcune idee che lei potrebbe avere sul trattamento della sua insufficienza renale.

Il modo per conoscere le idee dei pazienti sulla loro malattia è comunicare e relazionarsi, per gli emodializzati la permanenza in reparto è lunga e di più volte a settimana: è un'opportunità che può essere colta dal curante per dialogare con i pazienti! Peraltro c'è un lavoro fatto all'Università Johns Hopkins che dice come il contatto frequente con i dializzati possa migliorare i risultati di salute (10). Il metodo per migliorare l'adesione dei pazienti alle terapie prende le mosse da più comunicazione e più relazione.

Indirizzo degli Autori:

Dr. Bruno Bertone

Via Robiolo 119

13825 Valle Mosso (BI)

bruno.bertone@virgilio.it 


\section{Bibliografia}

1. M.A. Diefenbach. Illness representations http://can cercontrol.cancer.gov/brp/constructs/illness_represen tations/illness_representations.pdf

2. V. Bellizzi. SIN 2003 - http://www.sin-italy.org/pdf/pa ziente/divulgazione/26_prur_uremico.pdf

3. de Quevedo Welter E, Hubner Frainer R, Maldotti A, et al. Evaluating the association between alterations in mineral metabolism and pruritus in hemodialysis patient. http://www.scielo.br/pdf/abd/v86n1/ en_v86n1a03.pdf

4. Kammerer J, Garry G, Hartigan M, Carter B, Erlich L. Adherence in Patients On Dialysis: Strategies for Success. Nephrol Nurs J 2007; 34(5): 479-86.

5. Griva, Myers, \& Newman, 2000; Hampson, 1997; Jopson \& Moss-Morris, 2003. In C. Fowler, L.S. Baas - Illness Representations in Patients with Chronic Kidney Disease on Maintenance Hemodialysis. Nephrol Nurs J 2006; 33(2).
6. Fowler C, Baas LS. Illness Representations in Patients with Chronic Kidney Disease on Maintenance Hemodialysis. Nephrol Nurs J 2006; 33(2).

7. Denhaerynck K, Manhaeve D, Dobbels F, Garzoni D, Nolte C, De Geest S. Prevalence and consequences of nonadherence to hemodialysis regimens. Am J Critical Care 2007; 16(3): 222-35.

8. Rifkin DE, Laws MB, Rao M, Balakrishnan VS, Sarnak MJ, Wilson IB. Medication adherence behavior and priorities among older adults with CKD: a semistructured interview study. Am J Kidney Dis 2010; 56(3): 439-46.

9. DESG Teaching Letter, numero 12 - Il controllo nel tempo della malattia cronica www.desg.org/index2. php?option $=$ com_docman\&task $=$ doc

10. Plantinga LC, Jaar BG, Fink NE, et al. Frequency of patient-physician contact in chronic kidney disease care and achievement of clinical performance targets. Int J Qual Health Care 2005; 17(2): 115-21. 